\title{
Aspartate Aminotransferase to Platelet Ratio Index
}

National Cancer Institute

\section{Source}

National Cancer Institute. Aspartate Aminotransferase to Platelet Ratio Index. NCI

Thesaurus. Code C156512.

A calculation that indicates the likely presence of liver cirrhosis and fibrosis, measured as the relative measurement of aspartate aminotransferase (AST) to AST upper limit of normal, divided by the platelet count, and multiplied by 100 . 